\title{
Les propriétés mécaniques des articulations et les aspects physiques de la lubrification
}

\author{
Mechanical properties of articulations \\ and physical aspects of articular lubrication
}

\begin{abstract}
Jean-Pierre Renaudeaux
Laboratoire de Mécanique Expérimentale des Fluides, Université Pierre et Marie Curie, Bâtiment 502, Campus Universitaire, 91405 Orsay
\end{abstract}

On estime que 50000 prothèses de hanche seront posées en France cette année et que ce nombre est en augmentation rapide. Environ 1000 opérés devront à nouveau subir une intervention à la suite de défaillances mécaniques des prothèses.

Ces deux statistiques montrent que la compréhension de la mécanique articulaire est un problème d'actualité.

\section{Description et fonctionnement de l'articulation}

Les grandes articulations humaines (hanche, genou, etc.) ont évidemment des formes géométriques différentes. Elles sont cependant constituées suivant le même modèle (Fig. 1) et comprennent les 3 éléments importants pour la lubrification : os, cartilage, synovie.

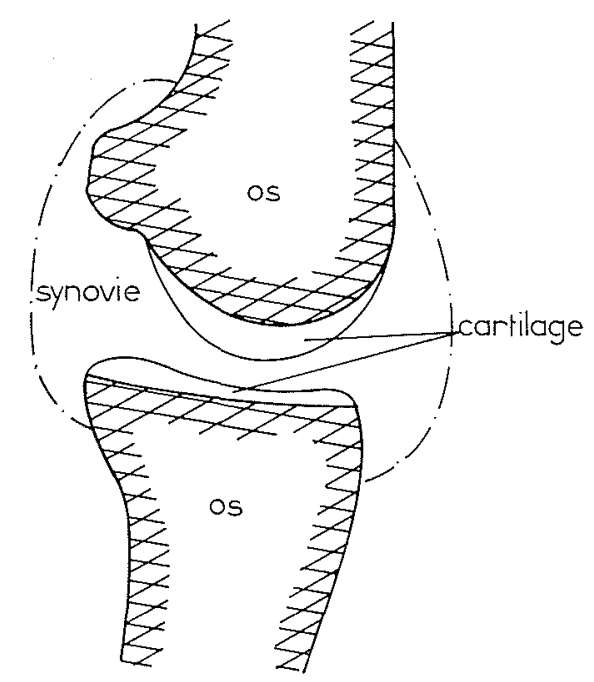

Figure 1 - Représentation schématique d'une articulation
L'étude dynamique à partir de plateaux de forces permet d'estimer les forces exercées au niveau des articulations porteuses. Paul [1] montre en 1969 que la force $P$ exercée sur l'articulation de la hanche peut atteindre au cours de la marche 7 fois le poids $W$ de l'homme (Fig. 2). La force exercée varie rapidement passant par deux maximum au cours d'un cycle. Comme il est admis que la surface de portance est de quelques $\mathrm{cm}^{2}$ il en résulte que la pression inter-cartilagineuse moyenne varie entre 0 et 30 bars. Il est à noter que les pressions ponctuelles peuvent etre plus importantes puisque Rumelhart [2] a atteint des pressions locales de 96 bars sur des essais de comportement de prothèses.

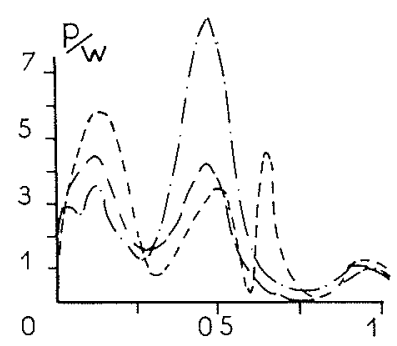

Figure 2 - Variation de la force appliquée au cours d'un cycle pour différents sujets masculins $-P$ : force appliquée $-W$ : poids du corps, en abscisse fraction du cycle. (d'après Paul).

Les composants de l'articulation, leurs propriétés mécaniques

Le fluide synovial

Il existe en très faible quantité dans l'articulation. Une ponction permet de retirer les quantités $<$ ou $=$ à $0,2 \mathrm{~cm}^{3}$. Il est composé de $95 \%$ d'eau, d'electrolytes en faible quantité (sodium, potassium, calcium) et d'une mucine : mucopolysaccharide acide non sulfaté composé d'acide hyaluronique et de protéines. 
Des mesures faites au viscosimètre Weissenberg par Ogston et Stanier en 1953 [3], Palfrey et White en 1968 [4], Davies et Palfrey en 1969 [5], Caygill et West en 1969 [6], Boyer, Bousquet et Bellet en 1976 [7], prouvent le comportement non-newtonien du fluide synovial. La figure 3 montre que, au repos le fluide se comporte comme un gel, et que pour des taux de cisaillement plus importants la viscosité peut tomber à quelques centipoises. Sur des sujets rhumatisants le liquide peut devenir pratiquement newtonien. D'après Caygill et West la viscosité $\mu$ peut s'écrire sous la forme

$$
\mu=\mu_{\infty}+\mathrm{AG}^{-2 / 3}
$$

avec $1 \mathrm{cp}<\mu_{\infty}<7 \mathrm{cp}$

$$
0,1<\mathrm{A}<2,3 \text { et } \mathrm{G} \text { en } \sec ^{-1}
$$

Le fluide synovial ne semble pas avoir de bonnes propriétés d'onctuosité.

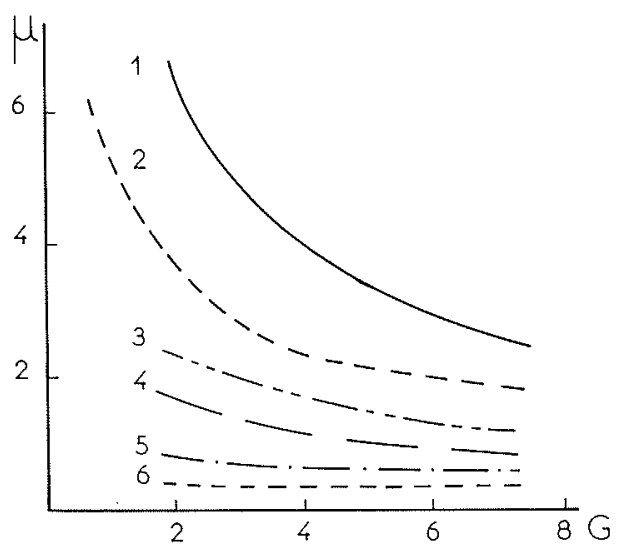

Figure 3 - Viscosité dynamique (en poiseuille) du fluide synovial en fonction du taux de cisaillement (en $\sec ^{-1} \times 10^{-2}$ ). (1), (2), (3) : synovies normales, (4) : synovie de rhumatisant, (5), (6) : synovies diluées de 50 à $75 \%$ (d'après Caygill)

\section{Le cartilage}

\section{Composition}

Il est constitué d'un assez petit nombre de cellules et d'une matrice extracellulaire abondante contenant une grande quantité d'eau: 75 à $78 \%$ du poids.

La matrice est composée d'un treillis de fibres collagène d'environ $600 \mu \mathrm{m}$ de diamètre et d'une substance non fibreuse de carbohydrates et de protéines; le tout peut être considéré comme un gel rigide.

\section{Structure}

L'étude au microscope électronique a permis de distinguer quatre zones. De la surface vers l'intérieur on rencontre successivement :

- la zone superficielle (5 à $10 \%$ de l'épaisseur) où les fibres de collagène sont parallèles entre elles et tangentes à la surface et où les cellules sont allongées (Fig. 4) ;

- la zone intermédiaire (40 à $50 \%$ de l'épaisseur) où les fibres de collagène sont entremêlées sans direction privilégiée et où les cellules sont sphériques (Fig. 5) ;

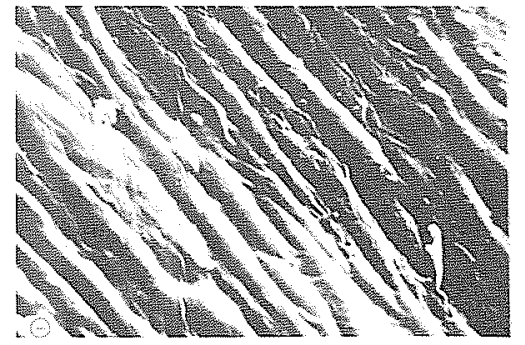

Figure 4 - Zone superficielle du cartilage (d'après Mc CALL)

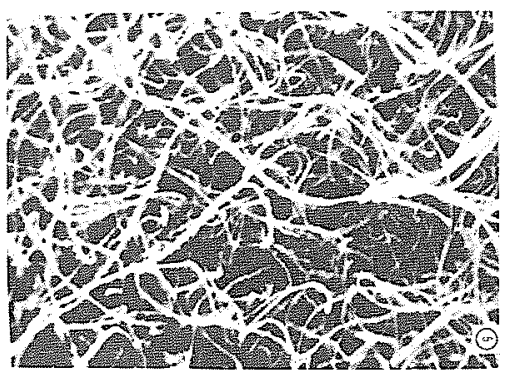

Figure 5 - Zone moyenne du cartilage (d'après Mc CALL)

- la zone profonde (40 à $45 \%$ de l'épaisseur), où les fibres deviennent plus nombreuses et commencent à prendre une orientation perpendiculaire à la surface ; - la zone calcifiée ( 5 à $10 \%$ de l'épaisseur) jouxtant l'os, qui comprend peu de cellules et est imprégnée de cristaux de sels de calcium.

\section{Surface du cartilage}

Si la structure de la matrice du cartilage est assez bien connue, de nombreuses controverses continuent à exister sur la nature des rugosités pouvant exister à sa surface.

Jusqu'à l'apparition du microscope électronique il était généralement admis que la surface cartilagineuse était lisse. Les premières études faites au microscope électronique à transmission confirmaient les résultats antérieurs. D'après Davies [8] en 1962 la rugosité est inférieure à $0,025 \mu \mathrm{m}$ et d'après Weiss [9] en 1968 les dépressions observées seraient inférieures à $0,3 \mu \mathrm{m}$. Le trop faible grossissement obtenu à l'aide d'un microscope à transmission a amené l'utilisation du microscope à balayage.

Mc Call [10] observe que la surface est rugueuse et parcourue par des fibres parallèles entre elles. Ce résultat semble confirmé par Walker [11] pour qui la surface est ondulée. Les sommets sont formés de larges fibres de collagène et les dépressions de réseaux de petites fibres. (Fig. 6)

I.C. Clarke [12] observe en 1972 ces mêmes rides, mais constate qu'elles n'existent que sur la périphérie des échantillons et déduit qu'elles sont la conséquence des dégradations faites pendant la préparation. Dans les autres régions il observe des dépressions ayant la forme de cratères (Fig. 7 et 8 ). Ces dépressions existent avec une densité de $430 / \mathrm{mm}^{2}$ et ont un diamètre de 20 à 

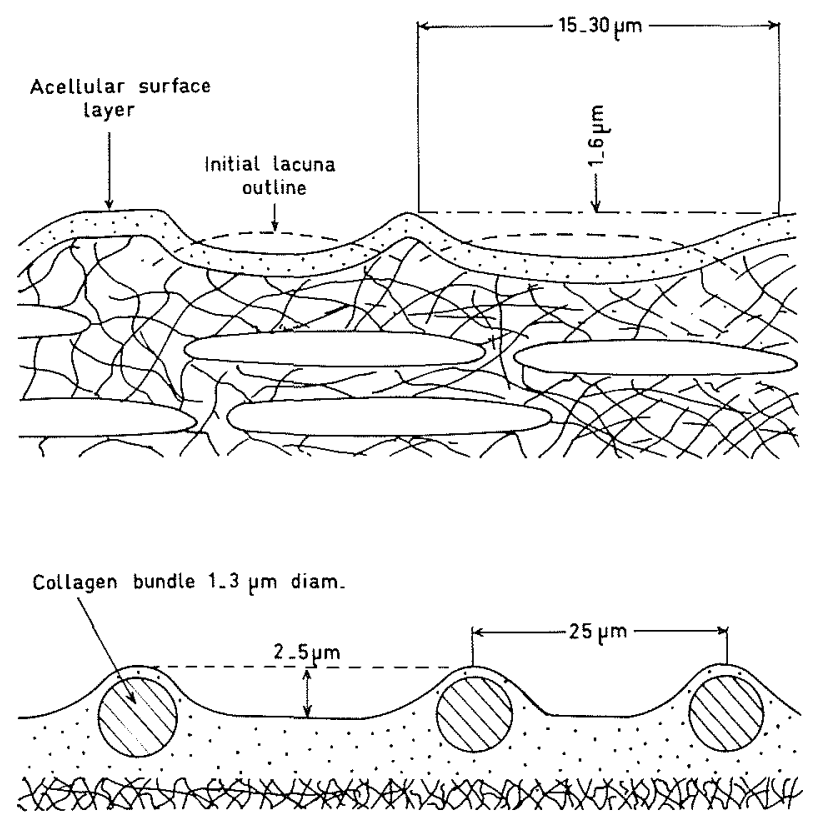

Figure 6 - Représentation schématique de la surface articulaire d'après des résultats de Walker

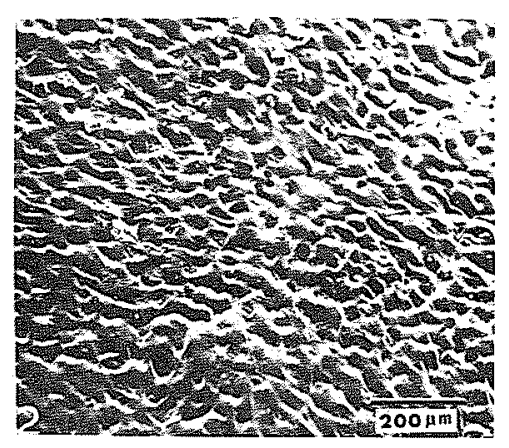

Figure 7 - Dépressions observées à la surface du cartilage (d'après I.C. Clarke) $(\times 100)$

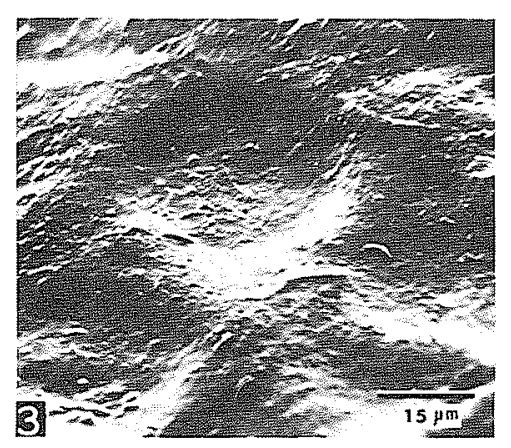

Figure 8 - Dépressions observées à la surface du cartilage (x 1530) d'après I.C. Clarke.

$40 \mu \mathrm{m}$. Elles sont donc comparables en taille et fréquence aux cellules sous jacentes. Pour savoir si ces creux sont dus au collabage des cellules pendant la préparation des échantillons ou s'ils existent aussi sur du cartilage frais, Clarke étudie à la lumière réfléchie du cartilage et au microscope à transmission des répliques de cartilage. Dans les deux cas il observe des contours semblables à ceux décrits ci-dessus. Sa con- clusion, très prudente, est la suivante : en l'absence de démonstration du contraire, il n'est pas irraisonnable d'assurer que la topographie décrite par le microscope à balayage est représentative de la surface articulaire pendant la vie.

\section{Propriétés mécaniques}

Les travaux de Kempson [13] et de Mc Cutchen [14] ont montré que le cartilage est un corps viscoélastique. Sous l'effet d'une compression constante, il se déforme pendant plus d'une heure.

Cette viscoélasticité a amené les auteurs à définir deux modules d'élasticité. Un module d'élasticité immédiat $E_{i}$ dû à la réponse instantanée des fibres et un module d'élasticité retardé dû à l'expulsion de l'eau. Les valeurs obtenues sont :

$$
\begin{array}{ll}
E_{i}=11,1 \times 10^{6} \mathrm{~N} / \mathrm{m}^{2} & \text { Mc Cutchen } \\
1,96 \times 10^{6}<E_{i}<14,4 \times 10^{6} \mathrm{~N} / \mathrm{m}^{2} & \text { Kempson } \\
E_{R}=0,32 \times 10^{6} \mathrm{~N} / \mathrm{m}^{2} & \text { Mc Cutchen } \\
E_{R}=E_{i} / 4 & \text { Kempson }
\end{array}
$$

Le module d'Young du cartilage est environ 1000 fois plus faible que celui de l'os. D'autre part, la rigidité dans les zones de contact est supérieure à celle existant dans la périphérie. Elle augmente avec la proportion de glycosaminoglycans, diminue lorsque la perméabilité augmente, mais aucune relation n'a été observée entre la rigidité et l'âge.

Kempson et Woo, Akeson et Jummot [15] ont fait des essais en traction figure 9 ; il en résulte que la rigidité décroît de la surface vers les couches profondes, dépend de la proportion de fibres de collagene et de leur orientation, mais elle est indépendante du pourcentage de protéoglycans. Le module d'Young étant fonction de la déformation, Woo reprend la relation proposée par Fung en 1967 :

$$
\sigma=A\left(e^{B \epsilon}-1\right)
$$

\section{Avec $A$ compris entre 1,1 et $3,3 \mathrm{MN} / \mathrm{m}^{2}$ \\ $B$ \\ 1,65 et 3,52}

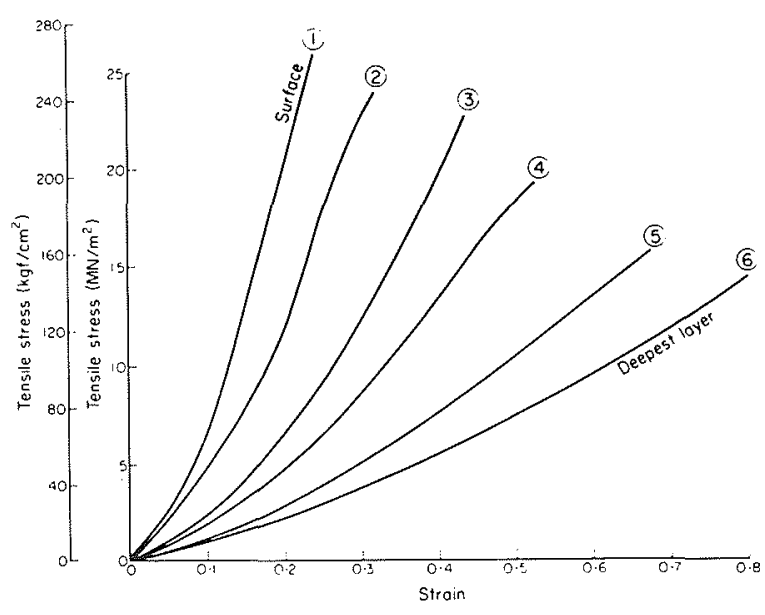

Figure 9 - Courbe de traction déformation du cartilage d'après G.E. Kempson. 
$A$ et $B$ dépendent de la profondeur et de l'orientation des échantillons. Les résultats semblent confirmer ceux obtenus avec le microscope à balayage sur l'existence d'une orientation privilégiée des fibres à la surface.

\section{La perméabilité du cartilage}

La perméabilité $K$ au cartilage est caractérisée par $K=\frac{L \mu}{S P} q_{\nu}$ (loi de Darcy).

avec $q_{v}$ : débit volumique

$S$ : section de l'échantillon

$\mu$ : viscosité

$L$ : longueur de l'échantillon

$P$ : différence de pression appliquée aux extrémités de l'échantillon.

Sous une différence de pression constante de 1 bar, Mc Cutchen trouve $K=5,8 \times 10^{-19} \mathrm{~m}^{2}$. Ses résultats sont confirmés par ceux de Maroudas [16]. Les recherches effectuées par A. Maroudas montrent en plus que la perméabilité décroît de la surface du cartilage $\left(3 \times 10^{-19} \mathrm{~m}^{2}<K\right.$ surface $\left.<9 \times 10^{-19} \mathrm{~m}^{2}\right)$ vers l'intérieur, $\left(10^{-19} \mathrm{~m}^{2}<K\right.$ près de l'os $\left.<2,5 \times 10^{-19} \mathrm{~m}^{2}\right)$, qu'elle ne varie pas systématiquement suivant la position de l'échantillon et qu'il n'y a pas de relation entre l'âge et la perméabilité.

Mulholland [17] étudiant l'influence de la pression sur la perméabilité trouve que, dans la gamme de ses essais, c'est-à-dire entre 0 et 6,9 bars, $K$ est indépendant de la différence de pression. Ces résultats sont infirmés par ceux de Mansour [18] qui observe une décroissance de la perméabilité lorsque la pression augmente. Ayant nous mêmes effectué des mesures à des pressions comprises entre 0,5 et 15 bars nous confirmons la décroissance observée par Mansour et nos résultats montrent

Figure 10 - Perméabilité du cartilage en fonction de la pression (en coordonnées Log-Log) que la relation existant entre la perméabilité et la différence de pression $P$ peut s'écrire :

$$
K=\frac{A}{P^{m}} \quad \text { et } m \text { de l'ordre de } 1 \text { (Fig. 10) }
$$

comme $q_{v}=\frac{K S}{\mu L}$ le débit passant à travers le cartilage est pratiquement indépendant de la pression appliquée.

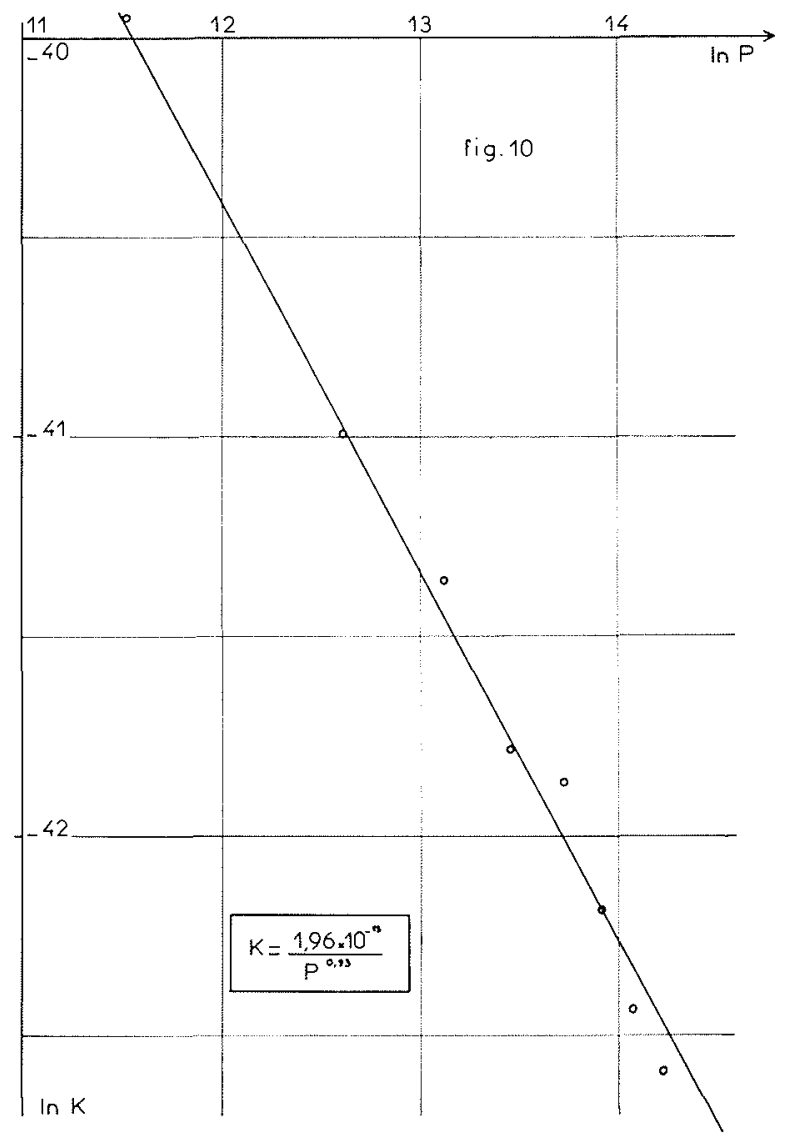

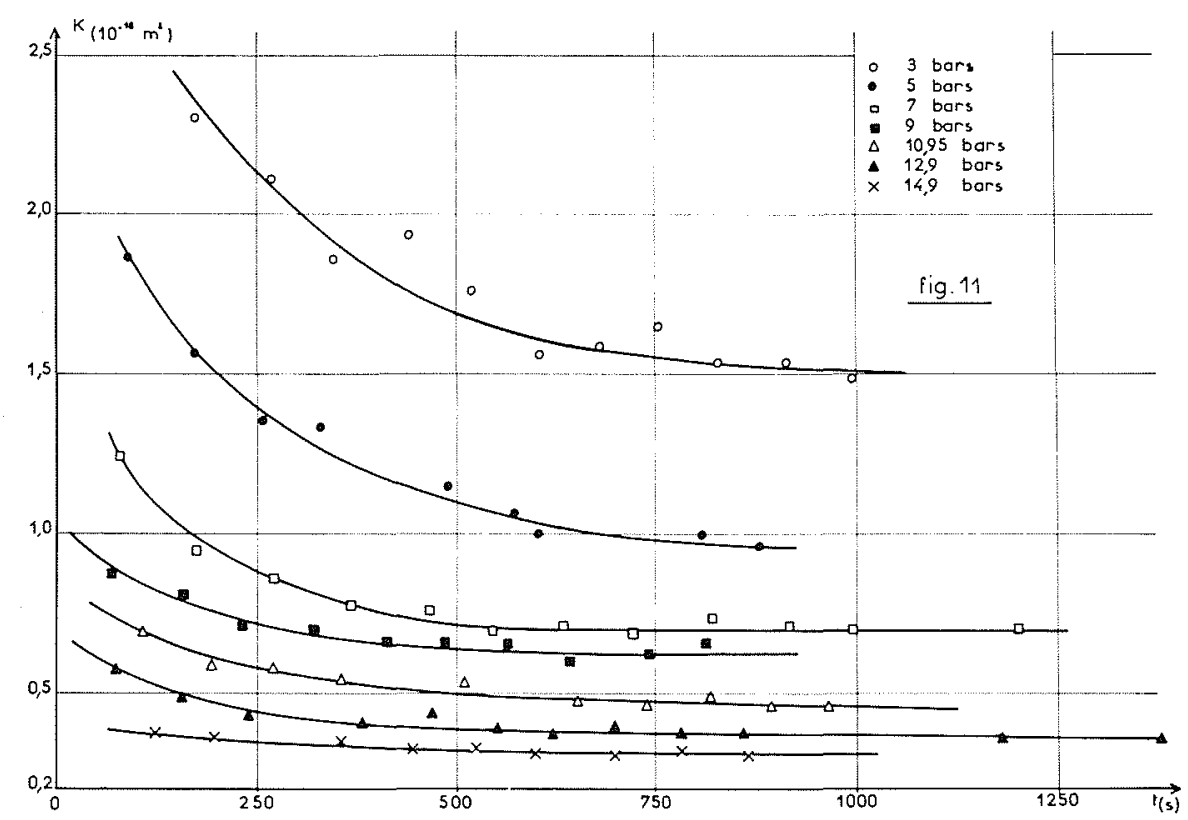

Figure 11 - Perméabilité du cartilage en fonction du temps pour différentes pressions. 
Tous les auteurs ont remarqué que le cartilage exsude sous l'effet de la pression et ont mesuré la perméabilité après environ 1 heure, temps nécessaire à la stabilisation. La perméabilité est donc une fonction du temps $K(t)$ et la marche étant dynamique il est intéres. sant de connaitre la loi $K(t)$. La figure 11 montre que la perméabilité décroît avec le temps, et obéit à la loi expérimentale $K(t)=B / t^{n}$ avec $B$ constant et $n$ de l'ordre de 0,2 pour $t$ compris entre $60 \mathrm{~s}$ et $1000 \mathrm{~s}$ gamme de nos expériences.

La porosité du cartilage calculée à partir de la perméabilité statique est de $64 \mathrm{~A}$. Cette dimension, inférieure à la dimension moléculaire de l'hyaluronate interdit à celui-ci de passer dans le cartilage.

\section{Aspects physiques de la lubrification articulaire}

Les mesures sur simulateur ou sur échantillon du coefficient de frottement ont donné des valeurs comprises entre 0,003 et 0,024 , valeurs très faibles compte tenu des conditions : à savoir très faible vitesse et très forte charge.

Une bibliographie extrêmement dense existe sur la tribologie des articulations et des prothèses articulaires (on pourrait probablement citer plus de 600 références).

\section{Lubrification hydrodynamique}

Mc Connaill écrit en 1950 que puisque le fluide est visqueux et d'épaisseur variable, la lubrification peut être du type hydrodynamique suivant l'effet du coin d'huile. Le calcul a montré depuis que l'application des lois de la lubrification hydrodynamique donnerait une épaisseur maximale du film de l'ordre de $130 \mathrm{~A}$ c'est-à-dire inférieure aux rugosités de parois.

\section{Lubrification élastohydrodynamique}

Les mêmes calculs obtenus à partir de la théorie élastohydrodynamique donnent une épaisseur de film de $15 \mu$ supérieure donc à la rugosité des parois et aux dimensions moléculaires de l'acide hyaluronique, Dowson [20].

\section{Lubrification limite}

Un certain nombre de chercheurs pensent que la lubrification limite est le mécanisme prédominant de la lubrification articulaire. Les raisons annoncées sont :

- le coefficient de frottement est faible même en l'absence de fluide synovial, Charnley [21];

- le coefficient de frottement décroit lorsque la force appliquée reste constante et la vitesse croît;

- il est indépendant de la viscosité du fluide. D'après les travaux de Linn [22], figure 12, il existe en effet

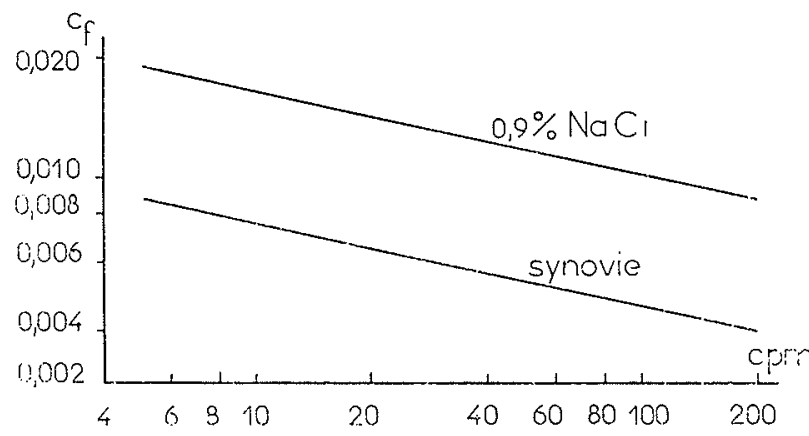

Figure 12 - Variation du coefficient de frottement avec la vitesse (d'après Linn)

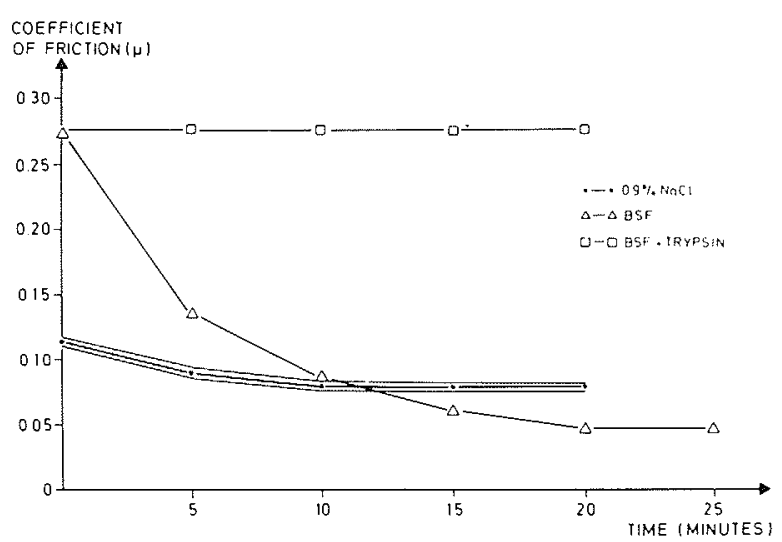

Figure 13 - Coefficient de frottement en fonction du temps, d'après I. Reimann.

un rapport constant entre le coefficient de frottement avec le sérum physiologique dont la viscosité est constante et le coefficient de frottement avec la synovie dont la viscosité décroît avec la vitesse:

- le coefficient de frottement décroît lorsque le temps augmente. Il faut un certain temps pour que de la synovie soit absorbée par le cartilage et joue un rôle de lubrifiant limite, Linn [22], Mc Cutchen [23] Reimann [24].

L'acide hyaluronique traité par hyaluronidase lubrifie pratiquement aussi bien que l'acide hyaluronique normal.

L'hyaluronidase cassant les molécules d'acide hyaluronique et rendant la synovie presque semblable à l'eau, la viscosité ne joue pas de rôle dans la lubrification (Linn) ;

- la synovie traitée par trypsine ne lubrifie pas aussi bien que la synovie normale (Reimann) et le coefficient de frottement d'une telle synovie est constant au cours du temps (Fig. 13);

La trypsine dissolvant les graisses, la mucine responsable de la lubrification serait composée de peptides et polypeptides, Swann [25], Little [26].

Deux raisons vont à l'encontre de cette théorie :

- les coefficients de frottement obtenus en régime de lubrification limite industrielle sont compris entre 0,05 et 0,1 , c'est-à-dire supérieurs à ceux de l'articulation; 
- la configuration moléculaire est un élément très important dans ce type de lubrification, or des polymères ayant une structure semblable à celle de l'acide hyaluronique ne lubrifient pas bien l'articulation.

\section{Squeeze film}

L'étude du mouvement a montré que les pressions sont variables au cours de la marche. A ces pressions variables doivent correspondre des épaisseurs de fluide fonction du temps. Est-ce que la résistance à l'écrasement (squeeze film) mise en évidence dans l'équation de Reynolds peut jouer un rôle dans la lubrification articulaire?

Fein [27] établit une formule théorique valable dans le cas des surfaces rugueuses. Après une seconde l'épaisseur du film est de 3,5 $\mu \mathrm{m}$ et après une heure elle est de $5,8 \times 10^{-2} \mu \mathrm{m}$ pour un fluide de viscosité $1 \mathrm{cp}$.

Les résultats obtenus sont en bon accord avec les expériences.

Le temps d'écrasement calculé par Fein qui est compatible avec les temps physiologiques est encore augmenté par d'autres facteurs :

- l'élasticité des parois (Higginson) [28]

- le caractère non newtonien de la synovie : très forte viscosité sous de faibles vitesses et formation d'agrégats d'hyaluronate à la surface du cartilage, Walter [11] ; Unsworth [29] mesure le coefficient de frottement d'une articulation en fonction du temps. Il observe que celui-ci décroît avec le temps sauf s'il applique la charge brusquement. Dans ce cas le coefficient de frottement augmente, passe par un maximum et décroit. Unsworth attribue la forme caractéristique de ces demières courbes à l'action du squeeze film (Fig. 14).
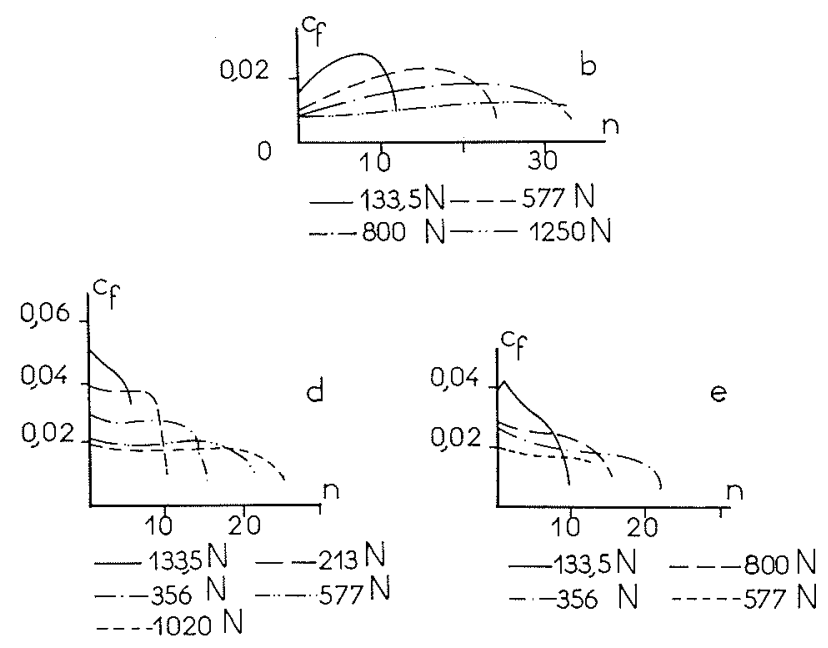

Figure 14 - Coefficient de frottement en fonction du temps d'après Unsworth

b) Articulation lubrifiée avec de la synovie : charge dynamique appliquée soudainement

d) Articulation non lubrifiée : charge appliquée soudainement e) Articulation lubrifiée avec de la synovie : charge statique
Théories faisant intervenir la perméabilité du cartilage

Comme nous l'avons vu plus haut le cartilage est perméable et viscoélastique et il est permis de penser que ces caractéristiques jouent un rôle dans la lubrification, cependant les deux facteurs (perméabilité et viscoélasticité) interviennent en sens inverse (Fig. 15). La perméabilité permet à l'eau de s'infiltrer dans le cartilage sous l'effet de la pression. A cause de sa viscoélasticité le cartilage se comprime et l'eau est expulsée.

Actuellement, aucune vérification n'a permis d'assurer quel phénomène l'emporte sur l'autre.

Mc Cutchen [14] pense que l'élasticité joue un rôle prédominant : le mécanisme qu'il propose et qu'il a appelé "weeping lubrication" ou lubrication par exsudation est le suivant (Fig. 16). La pression fait sourdre l'eau et les petites molécules du cartilage qui se déforme, emprisonnant entre ses surfaces le fluide sous pression ainsi libéré. Les bords de la zone de charge sont maintenus par l'affrontement de deux courants liquides,

1. PERMEABILITE

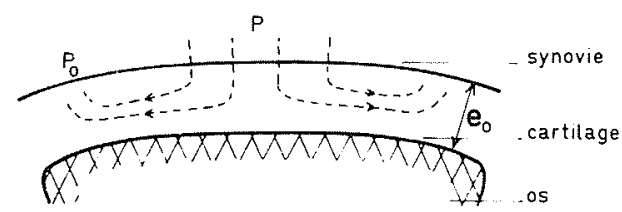

2. VISCOE LASTICITE

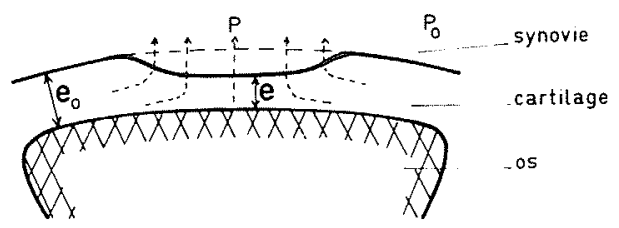

Figure 15 - Rôle de la perméabilité et de l'élasticité du cartilage dans la lubrification.

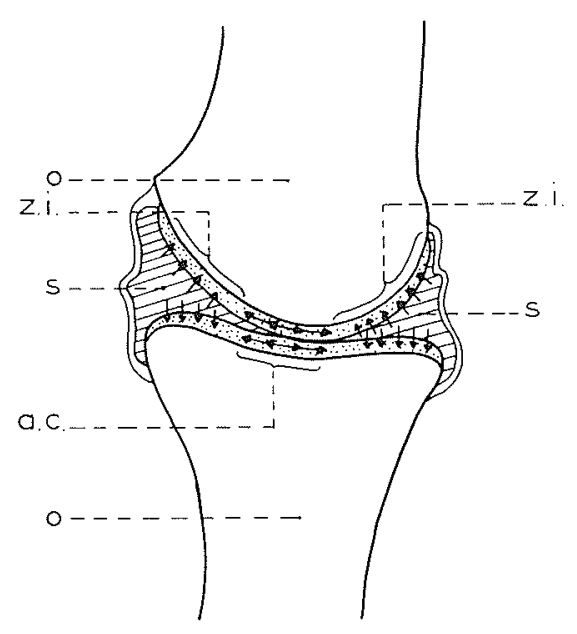

Figure 16 - La "Weeping lubrication" d'après Mac Cutchen 


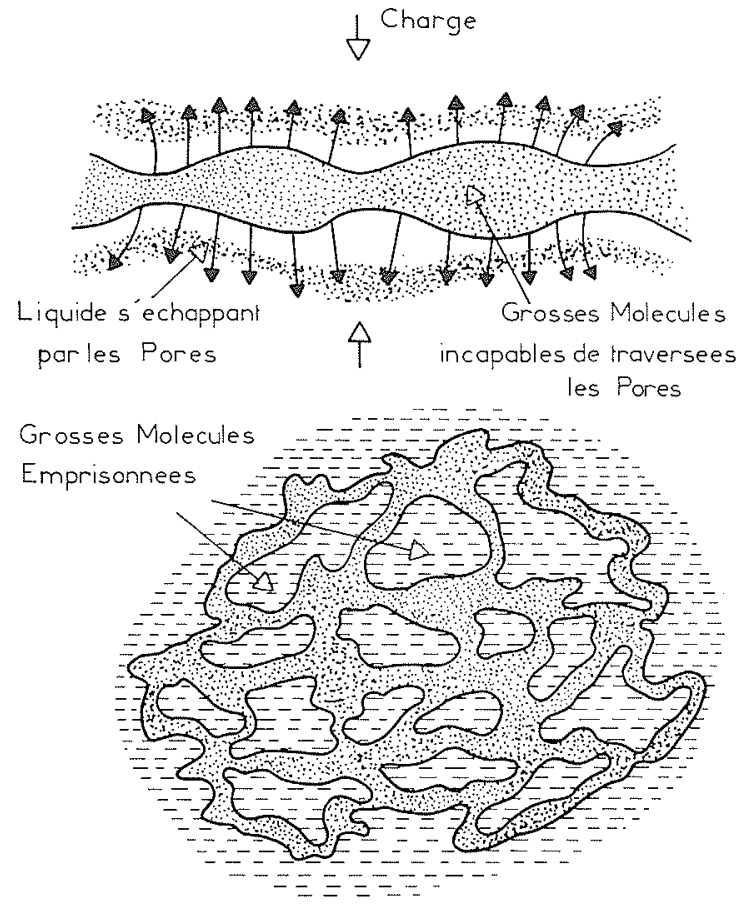

Figure 17 - La "Boosted lubrication" d'après Dowson.

celui qui a tendance à chasser le fluide vers l'extérieur de la zone sous pression et celui qui, dans le cartilage, a tendance à faire sortir l'eau.

Dowson [30] pense au contraire que la perméabilité est essentielle. Son hypothèse qu'il appelle "boosted lubrication" ou lubrification par filtration est celle-ci : (Fig. 17) sous l'effet de la pression, le fluide synovial s'échappe par les surfaces latérales, l'eau et les petites molécules passent à travers le cartilage. Il en résulte une augmentation de la concentration d'acide hyaluronique et la formation d'un gel prisonnier des irrégularités de surface du cartilage.

\section{Conclusion}

"Aucune théorie valable dans l'ingéniérie n'est assez sophistiquée pour expliquer totalement le mouvement des articulations, mais l'approche la plus réaliste est la théorie élastohydrodynamique dans laquelle il faut prendre en compte d'autres phénomènes tels que les déformations latérales ou le mouvement intermittent". (Dintenfass 1963). Cette phrase déjà ancienne, de Dintenfass, est encore en partie d'actualité. Il est en effet probable que, au cours de la marche, plusieurs mécanismes interviennent simultanément ou successivement, mais les connaissances sur les caractéristiques physiques et mécaniques des matériaux, les conditions réelles d'utilisation ne sont pas encore assez sures pour pouvoir expliquer de façon rigoureuse les mécanismes régissant réellement la lubrification articulaire.
Bibliographie

[1] PAUL (1969) - Magnitude of forces transmitted at hip and knee-joints in 'Lubrication and wear in joints" Wright ed. 1969.

[2] RUMELHART - Communication personnelle

[3] OGSTON A.G. and STANIER J.E. (1953) - The physiological function of hyaluronic acid in synovial fluids : viscous, elastic and lubricant properties J. Physio., pp. 119,244

[4] PALFREY and WHITE (1968) - The viscosity of synovial fluid during oscillatory movement. Biorheology, vol. 5-3-sept. pp. 189-198.

[5] DAVIES and PALFREY (1969) - Physical properties of synovial fluid in "Lubrication and wear in joints" Wright ed.

[6] J.C. CAYGILL, G.H. WEST - The rheological hehaviour of synovial fluid and its possible relation to joint lubrication. Med. Biol. Engng, vol. 7-1969-pp. 507-516.

[7] BOYER, BOUSQUET et BELLET - au 1er Congrès de biomécanique, Lyon, Juin 1976.

[8] DAVIES D.V., BARNETT CH, COCHRONE W. PALFREY A.J. (1962) - Electron microscopy of articular cartilage in the young adult rabbit. Ann. Rheum. Diseas 21-11.

[9] WEISS C., ROSENBERG L., HELFET AJ. (1968) - An ultrastructural study of normal young adult human cartilage J. Bone Joint Surg., vol. 50 A p. 663.

[10] J.Mc CALL - Load deformation response of the microstructure of articular cartilage in "Lubrication and wear in joints", Wright ed. (1969)

[11] WALKER P.S., SIKORSKI J., DOWSON D., LONGFIELD MD., WRIGHT V., BUCKLEY T. - Behaviour of synovial fluid on surfaces of articular cartilage. A. Scanning Electron Microscope Study. Ann. Rheum. Diseases 1969, $28,1,14$.

[12] CLARKE IC. - The Microevaluation of Articular Surface Contours - Annals of Biomedical Engineering, 1, 1972 pp. $31-43$

[13] KEMPSON G.E. - Mechanical properties of articular cartilage in adult articular cartilage - edited by A.R. Freeman, Pitman Medical 1973.

[14] Mac CUTCHEN C.W. - The frictional properties of animal joints wear 1962-5 pp. 1-17.

[15] WOO S.L.Y., AKESON W.H., JEMMOTT G.F. - Measurements of non homogeneous directionnal mechanical properties of articular cartilage in tension. J. Biomech., 1976 vol. 9 pp. $785-791$.

[16] MAROUDAS A. - Physico chemical properties of articular cartilage in adult articular cartilage, ed. by A.R. Freeman (1973)

[17] MULHOLLAND R. - Lateral hydraulic permeability and morphology of articular cartilage.

[18] MANSOUR J.M., MOW V.C. - The permeability of articular cartilage under compressive strain at high pressures - J. Bone Joint Surg. vol. $58 \mathrm{n}^{\circ} 4$ pp. 509-516.

[19] Mac CONNAILL M.A. - The movements of Bones and joints. - J. Bone Joint Surgery 32-3- mai 1950. pp. 244252.

[20] DOWSON D. - Lubrication in human joints In "Lubrication and wear in joints" - Wright ed.

[21] J. CHARNLEY - The lubrication of animal joints in Symposium of Biomechanics pp. 12-22 - London Institution of Mechanical Engng.

[22] LINN F.C. - Lubrication of animal joints. J. Bone Joint Surg. septembre 1967 vol. $49 \mathrm{n}^{\circ} 6$ pp. 1078-1096.

[23] Mac CUTCHEN C.W. - Boundary lubrication by synovial fluid. Demonstration and possible osmotic explanation. Federation proceedings 1966 vol. 25 pp. 1061-1068. 
[24] REIMANN I., STROUGAARD J., NORTHEVED A., JOHNSEN S.J. - Demonstration of boundary lubrication by synovial fluid. Acta. Orthop. Scand. 46-1975 pp 1-10.

[25] SWANN D.A., RADIN EL, NAZIMIEC N. - Role of hyaluronic acid in joint lubrication. Annals of Rheumatic Diseases - vol. $33 \mathrm{n}^{\circ} 4$ - July 74 pp. 318-326.

[26] LITTLE T., FREEMAN A.R., SWANSON A. - Experiments of friction in the human hip joint in "Lubrication and wear in joints" Wright ed.

[27] FEIN R.S. (1966-67) - Are synovial joints squeeze film lubricated. Proc. int. Mech. Engng., 8, 125-128.
[28] HIGGINSON G.R., NORMAN R. (1974), - A model investigation of squeeze film lubrication in animal joints. Phys. Med. Biol., 19 pp. 785-792.

[29] UNSWORTH A., DOWSON D., WRIGHT W. (1974) The frictional behaviour of synovial joints. $J$. Lubrication Technology. Trans. ASME LUB 38 and 39.

[30] DOWSON D., UNSWORTH A., VRIGHT V. (1970) Analysis of "boosted lubrication" in human joints. $J$. mech. Engng. Sc., 12 pp. 364-369.

[31] DINTENFASS L. (1963) - Lubrication in Synovial joints : a theoretical analysis. $-J$. Bone joint Surgery, 45 pp. $124 \mathrm{i}-1256$. 